\title{
2019-nCoV 3C-Like Protease carries an activity-enhancing T285 /A variation which may contribute to its high infectivity
}

\author{
Mei Dang and Jianxing Song* \\ Department of Biological Sciences, Faculty of Science, National University of Singapore
}

Running title: 2019-nCoV 3CLpro carries an activity-enhancing variation

*Correspondence author. Email: $\underline{\text { dbssjx @nus.edu.sg }}$

Key words: 2019-nCoV; 3C-like protease; Enzymatic catalysis; T285A variation. 


\begin{abstract}
In 2019, a mysterious outbreak of pneumonia occurred in Wuhan, China, which then rapidly spread over the world. Now it was identified to be associated with a new coronavirus "2019-nCoV". Noticeably, 2019-nCoV appears to have much higher infectivity than SARS-CoV but the underlying mechanisms still remain unknown. Here, we found that despite the relatively low identity at nucleotide level, the 2019-nCoV and SARS CoV have almost identical 3C-like proteases in both sequence and structure. Most unexpectedly, the 2019-nCoV 3C-like protease carries the Thr285Ala variation we previously constructed and showed to enhance the enzymatic activity. This finding may partly explain its observed high infectivity and certainly needs to be studied experimentally and computationally.
\end{abstract}




\section{Introduction}

In 2019, a mysterious outbreak of pneumonia occurred in Wuhan, China which then rapidly spread over the world (https://www.who.int/westernpacific/emergencies/novelcoronavirus). This pneumonia was now identified to be caused by a novel coronavirus named as "2019-nCoV" (Zhou et al., 2020). Noticeably, based on the available data, the 2019-nCoV appears to have much higher infectious ability even than another coronavirus leading to the outbreak of severe acute respiratory syndrome (SARS) in 2002. So a key question arises what unique properties of 2019-nCoV account for its high infectivity. To understand the underlying mechanisms for its high infectivity is essential for developing effective strategies to fight against 2019-nCoV.

Like the coronaviruses causing SARS (SARS-CoV) and Middle East respiratory syndrome coronavirus (MERS-CoV), 2019-nCoV is also enveloped, positive-stranded RNA viruses with the largest single-stranded RNA genome (27-31 kilobases) (Zhou et al., 2020). The large replicase gene encodes two viral polyproteins, namely pp1a and pplab, which have to be processed into active subunits for genome replication and transcription by viral proteases including the 3C-Like protease (3CLpro), also known as main protease (Mpro). As a consequence, SARS 3CLpro has been extensively characterized to be a key target for development of antiviral therapies (Anand et al., 2002; Yang et al., 2003; Shi et al., 2004; Shi et al., 2008; Shi et al., 2011; Lim et al., 2014; Lim et al., 2010).

The coronavirus 3CLpro is so named to reflect the similarity of its catalytic machinery to that of the picornavirus 3C proteases (Allaire et al., 1994). Noticeably, both 3C and 3CL-Like proteases utilize the two-domain chymotrypsin fold to host the complete catalytic machinery, 
which is located in the cleft between domains I and II. Intriguingly, however, in the coronavirus 3CLpro, a 100-residue helical domain was evolutionarily acquired at its C-terminus. Moreover, unlike 3C protease, only the homodimeric form is catalytically competent for the CoV 3CLpro. After intense studies, now it has been clear that both chymotrypsin fold and extra domain are critical for dimerization (Anand et al., 2002; Yang et al., 2003; Bacha et al., 2004; Shi et al., 2004; Chen et al., 2006; Chen et al., 2008; Shi et al., 2008; Shi et al., 2011; Lim et al., 2014; Lim et al., 2010).

We have been particularly focused on understanding the role of the extra domain and subsequently revealed that although the catalytic fold and extra domain could fold independently, the catalytic fold alone was monomeric but almost inactive (Shi et al., 2004). Therefore, we further conducted a systematic mutagenesis study which led to identification of the extra-domain residues critical for both dimerization and catalysis (Shi et al., 2006). Interestingly, we found that the residues important for catalysis and dimerization constitute a nano-channel, which are composed of residues from both catalytic and extra domains. Moreover, we determined the highresolution structure of R298A, a monomeric mutant triggered by a point mutation on the extra domain, in which the most radical changes have been found within the catalytic machinery (Shi et al., 2008). Very unexpectedly, we previously also found that the extra-domain residues S284T285-I286 play a key role in modulating the catalytic machinery and the mutation of each residue would result in the significant enhancement of the activity (Shi et al., 2006). In particular, the mutant with all three residues mutated to Ala led to a 3.6-fold enhancement of the catalytic activity but only slightly enhanced dimerization (Shi et al., 2006). Surprisingly, our determination of its crystal structure reveals that STI/A still adopts the dimeric structure almost identical to that of the wild-type (WT), only with a slightly change of the extra-domain packing 
(Lim et al., 2014). As a consequence, we conducted molecular dynamics (MD) simulations for both WT and STI/A and remarkably, the most dramatic changes in STI/A simulations are associated with the nano-channel we previously identified (Shi et al., 2006). This change ultimately led to the enhanced dynamic stability of the key components composed of the catalytic machinery, thus rationalizing its enhance activity (Lim et al., 2014).

In the present study, we first identified the 3C-like protease of 2019-nCoV and subsequently built up a homology model for it. Most unbelievably, we found that the 3C-like protease of 2019-nCoV carries the Thr2885Ala variation which we previously constructed and found to enhance the catalytic activity. As the $3 \mathrm{C}$-like protease is essential for the replication of the virus, we thus propose here that this variation may partly contribute to its high infectivity. 


\section{Results and Discussion}

\section{The 3C-like proteases of SARS-CoV and 2019-nCoV have very high sequence identity}

Here we first identified the sequence of the $3 \mathrm{C}$-like proteases of $2019-\mathrm{nCoV}$ from its genomic data and aligned with those of several other coronaviruses which include those isolated from a bat causing MERS (St John et. al., 2015), and another bat (RaTG13) which was reported to be collected in Yunnan 2013 but with its genomic data just deposited on Jan 27, 2020 (Zhou et al., 2020).



\section{Figure 1. Sequence alignment}

Sequence alignment of the 3C-like proteases of several coronaviruses including from a bat causing MERS with the crystal structure (4YOJ), SARS, another bat (RaTG13) and 2019-nCoV. 
As shown on Fig. 1, the sequence of the 3C-like protease of 2019-nCoV has very low identify $(\sim 50 \%)$ to that of the MERS-CoV which was also isolated from a bat. Very unexpectedly, despite a relatively-low identity of the genomic sequence to that of SARS-CoV (79.5\%) (Zhou et al., 2020), as seen in Fig. 2A, identity of the 3C-like protease sequences of 2019-nCoV and SARS-CoV are extremely high ( 96\%). Most unbelievably, the 3C-like protease sequences of 2019-nCoV and RaTG13-CoV only have one-residue difference with identity of $99.7 \%$, thus implying that they might evolve from the same CoV strain very recently.

A
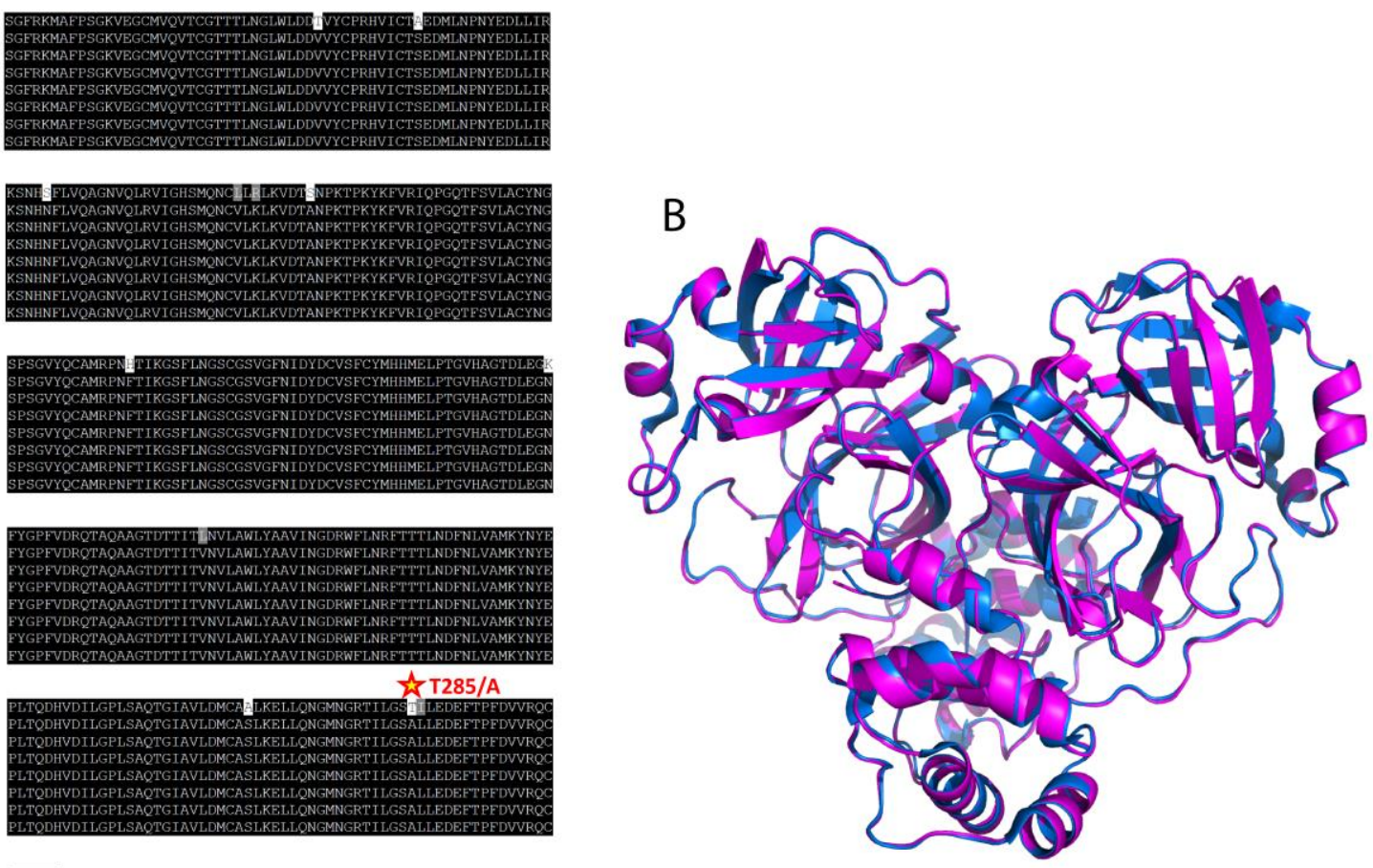

त T285/A
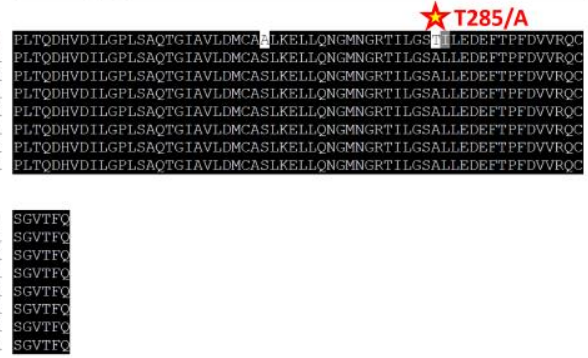

\section{Figure 2. Sequence and structure}

(A) Sequence alignment of the 3C-like proteases of SARS-CoV and different isolates of 2019nCoV. (B) Superimposition of the crystal structure of the 3C-like proteases of SARS (blue) and the homology modeling model of the 3C-like proteases of 2019-nCoV (purple). 


\section{The 3C-like proteases of 2019-nCoV and SARS-CoV have high similar structures}

As they have the extremely-high sequence identity, we constructed the structure model of the 3C-like proteases of 2019-nCoV by use of the crystal structure of the STI/A mutant of the SARS 3C-like protease we previously determined (PDB ID of 3EA8) which has no extra residue from any fusion tag (Lim et al., 2014). Indeed, the 3C-like proteases of 2019-nCoV has a very similar structure to that of the SARS protease, with a backbone RMSD value of only $0.8 \AA$ (Fig. 2A).
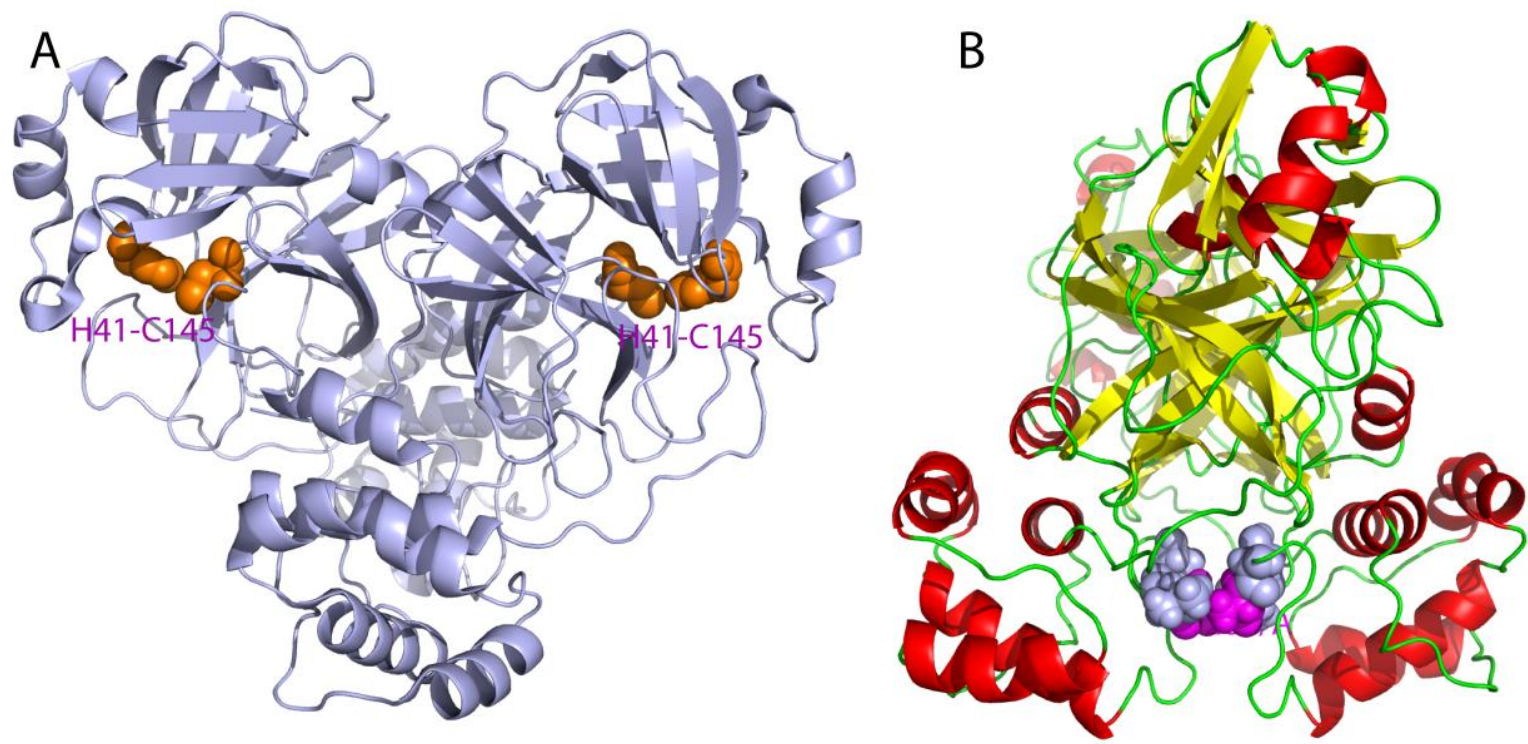

Figure 3. The 2019-nCoV 3C-like proteases carries an activity-enhancing T285A variation (A) The homology modeling model of the 3C-like proteases of $2019-\mathrm{nCoV}$ with the catalytic dyad His41-Cys145 displayed in spheres. (B) The homology modeling model of the 3C-like proteases of 2019-nCoV with the Ser284-Thr285-L286 displayed in spheres and Thr285 colored in purple.

As also seen in Fig. 3A, the catalytic dyad Cys41-Cys145 is also highly similar to that of the SARS protease. Most interestingly, over the Ser284-Thr285-Ile286 region of the SARS protease, which is located on the extra domain and far away from the catalytic dyad, the residue 
Thr285 of the 2019-nCoV 3C-like protease was replaced by Ala. Based on our previous results on the SARS 3C-like protease (Shi et al., 2006), this variation is expected to enhance its catalytic activity, thus more effectively processing the polyproteins into the active subunits for the viral replication. Therefore, the Thr285Ala variation may partly contributes to the high infectivity of the 2019-nCoV. 


\section{Conclusion}

Here, we found that despite the relatively low identity at nucleotide level, the 2019-nCoV and SARS CoV have almost identical 3C-like proteases in both sequence and structure. Most unexpectedly, the 2019-nCoV 3C-like protease carries the Thr285Ala variation we previously constructed and showed to enhance the enzymatic activity. This finding may partly rationalize its observed high infectivity and certainly demands further studies by experimental and computational approaches.

\section{Acknowledgement}

This study is supported by Ministry of Education of Singapore (MOE) Tier 2 Grant MOE2015-T2-1-111 to Jianxing Song. 


\section{References}

Allaire, M., M. M. Chernaia, B. A. Malcolm, and M. N. James. (1994). Picornaviral 3C cysteine proteinases have a fold similar to chymotrypsin-like serine proteinases. Nature 369:72-6.

Anand, K et al., (2002). Structure of coronavirus main proteinase reveals combination of a chymotrypsin fold with an extra alpha-helical domain. EMBO J 21:3213-24.

Bacha U, Barrila J, Velazquez-Campoy A, Leavitt SA, Freire E. (2004) Identification of novel inhibitors of the SARS coronavirus main protease 3CLpro. Biochemistry. 43:4906-12.

Chen, H., P. Wei, C. Huang, L. Tan, Y. Liu, and L. Lai. (2006). Only one protomer is active in the dimer of SARS 3C-like proteinase. J Biol Chem. 281:13894-8.

Chen, S., Hu, T., Zhang, J., Chen, J., Chen, K., et al., 2008. Mutation of Gly-11 on the dimer interface results in the complete crystallographic dimer dissociation of severe acute respiratory syndrome coronavirus 3C-like protease: crystal structure with molecular dynamics simulations. J. Biol. Chem. 283, 554e564.

Lim L, Shi J, Mu Y, Song J. Dynamically-driven enhancement of the catalytic machinery of the SARS 3C-like protease by the S284-T285-I286/A mutations on the extra domain. PLoS One. 2014 Jul 18;9(7):e101941.

Lim L, Gupta G, Roy A, Kang J, Srivastava S, Shi J, Song J. Structurally- and dynamicallydriven allostery of the chymotrypsin-like proteases of SARS, Dengue and Zika viruses. Prog Biophys Mol Biol. 2019 May;143:52-66.

Shi, J., Z. Wei, and J. Song. (2004). Dissection study on the severe acute respiratory syndrome 3C-like protease reveals the critical role of the extra domain in dimerization of the enzyme: defining the extra domain as a new target for design of highly specific protease inhibitors. J Biol Chem 279:24765-73. 
Shi, J., and J. Song. (2006). The catalysis of the SARS 3C-like protease is under extensive regulation by its extra domain. FEBS J 273:1035-45.

Shi, J., J. Sivaraman, and J. Song. (2008). Mechanism for controlling the dimer-monomer switch and coupling dimerization to catalysis of the severe acute respiratory syndrome coronavirus $3 \mathrm{C}$ like protease. J Virol 82:4620-9.

Shi J, Han N, Lim L, Lua S, Sivaraman J, Wang L, Mu Y, Song J. (2011) Dynamically-driven inactivation of the catalytic machinery of the SARS 3C-like protease by the N214A mutation on the extra domain. PLoS Comput Biol. 7:e1001084.

St John, S.E., Tomar, S., Stauffer, S.R., Mesecar, A.D. Targeting zoonotic viruses: Structurebased inhibition of the 3C-like protease from bat coronavirus HKU4-The likely reservoir host to the human coronavirus that causes Middle East Respiratory Syndrome (MERS). (2015) Bioorg.Med.Chem. 23: 6036-6048

Yang, H., M. Yang, Y. Ding, Y. Liu, Z. Lou, Z. Zhou, L. Sun, L. Mo, S. Ye, H. Pang, G. F. Gao, K. Anand, M. Bartlam, R. Hilgenfeld, and Z. Rao. (2003). The crystal structures of severe acute respiratory syndrome virus main protease and its complex with an inhibitor. Proc Natl Acad Sci U S A 100:13190-5.

Zhou P. et al., A pneumonia outbreak associated with a new coronavirus of probable bat origin. Nature. 2020 Feb 3. doi: 10.1038/s41586-020-2012-7. 